\title{
Application of Particulate Matter Analysis Performed by SEM-EDS to Air Quality Studies
}

\author{
R. Ramirez-Leal,* M. Valle-Martinez* and A. Acosta-Betancourt* \\ *Centro de Estudios Superiores del Estado de Sonora, Ley federal del Trabajo Final s/n Colonia Apolo. \\ Hermosillo, Sonora, Mexico C.P. 83100, Escuela de Ecologia.
}

This work describes the use of Scanning Electron Microscopy (SEM) combined with X-ray Dispersive Energy Spectrometry (EDS) to characterize of inorganic atmospheric particles samples collected on TSP and PM10 filters from January 2009 through December 2009 from three zones within the city of Hermosillo, Sonora. Specimens were initially processed by separating the collected particles from the filters by means of submersing a $2 \mathrm{~cm}^{2}$ section of each filter into isopropilic alcohol within a test tube for 5 minutes. Then, an aliquot of the suspension was placed over a sample holder and into the SEM

Particulate matter in the air includes total suspended particles (TSP), particulate matter with median aerodynamic diameter less than $10 \mu \mathrm{m}$ (PM10). Suspended Particulate Matter is a main pollutants and it may become a problem in autumn and winter because of the topography of the city and meteorological conditions. The harmful effects of aerosols depends on mass concentrations of airborne particulate matter, but also on certains characteristics such as the chemical composition and microphysical properties of the constituting individual particles. Thus, a good knowledge of physico-chemical characteristics of atmospheric particles is essential. In urban and industrial environments, physical processes like condensation or coagulation and heterogeneous reactions of particles can occur rapidly in polluted air masses.

Sampling of aerosols generally takes several hours to collect enough particulate matter for subsequent chemical analysis. Unlike many bulk techniques, single particle analysis by scanning electron microscopy combined with X-ray Dispersive Energy Spectrometry requires light sample loadings can provide information on the size distribution and chemical composition of pollution aerosols, notably in source areas where reactive compounds are present.

The results obtained from the characterization of the particles captured by TSP and PM10 filters, showed that elements such as $\mathrm{Al}, \mathrm{Si}, \mathrm{Ca}, \mathrm{Fe}, \mathrm{K}$, and $\mathrm{Mg}$ (fig.1) are largely abundant, and are usually related with litophilic sources, while elements such as $\mathrm{Cl}$ are more frequently associated with incineration and combustion activities as well as derived from marine aerosols.

The chemical analyses performed on the aerosol forming conglomerates allow us to determine air quality on each city. However, these might ignore the chemical elements that occur in smaller amounts because these are not considered or detected.

Given that there is relatively little knowledge on speciation and size of atmospheric particles, the elements found among the atmospheric particles caught on the TSP and PM10 filters highlight the importance of individual characterization of atmospheric aerosol. This knowledge is valuable additional information about the possible actions and potential effects that air pollutants, of natural or anthropogenic origins, have within the studied area. 


\section{References}

[2]Berico, M., Luciani, A. and Formignani, M. 2007. Atmospheric aerosol in an urbana areameasurement of tsp and pm10 standards and pulmonary deposition assessment. Atmospheric Environment 31(21): 3659-3665.

[2]Giugliano, M., Lonati, G., Butelli, P., Romele, L. and Grosso, M. 2005. Fine particle (pm2.5pm1) at urban sites with different traffic exposure.Atmospheric Environment 39: 2421-2431

[2]Hueeglin,Ch., Gehrig, R., Baltensperger, U., Gysel, M.and Vonmont, H. 2005. Chemical characterization of pm2.5, pm10 and coarse particles at urban, near-city and rural sites in Switzerland. Atmospheric Environment 39: 637-651

[2] J.I. Goldstein et al., Scanning Electron Microscopy and X-ray Microanalysis, Plenum, New York, 1992.

a)

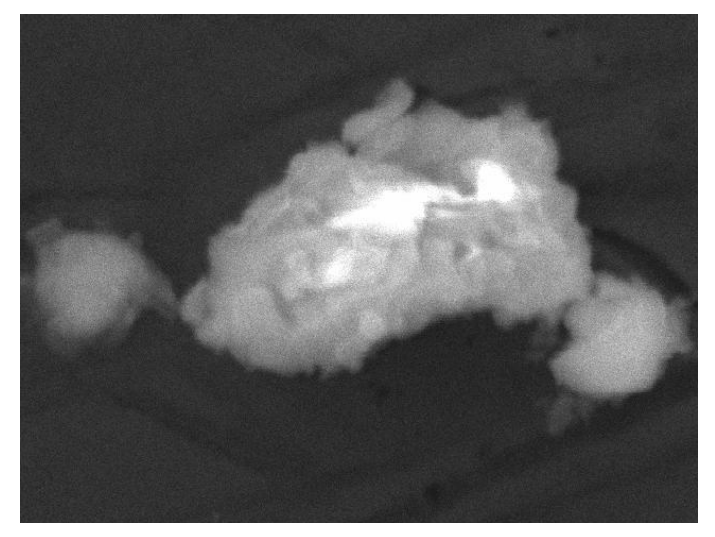

b)

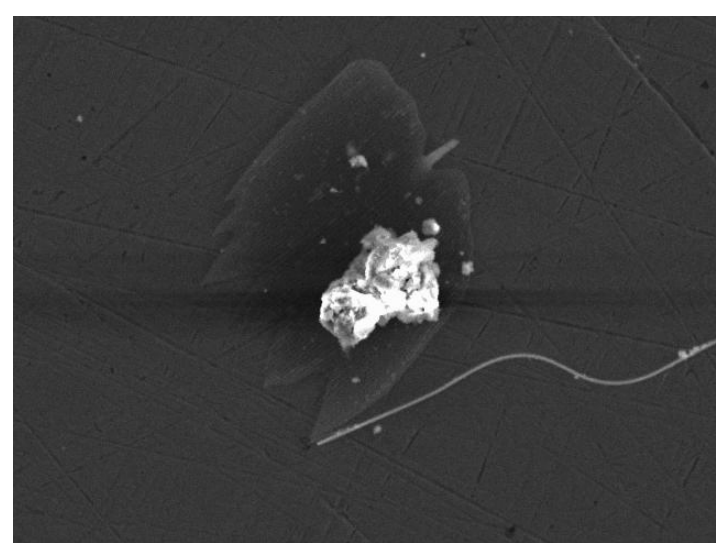

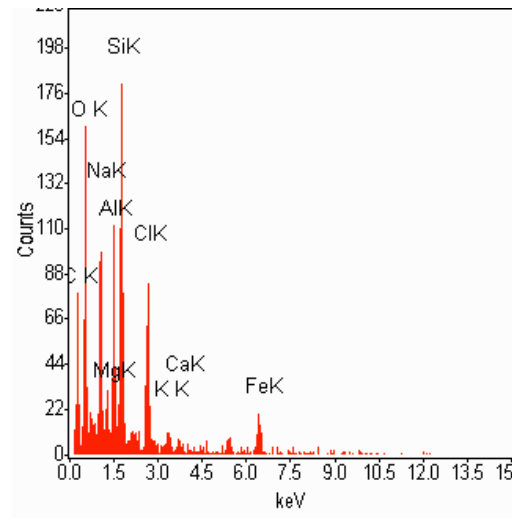

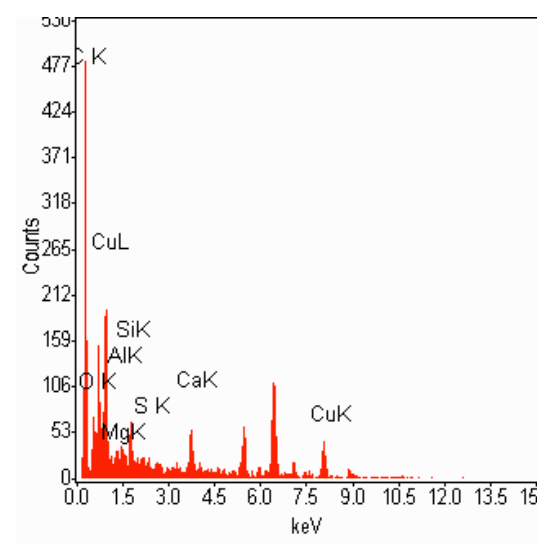

Fig.1.- Image and chemical composition of (a)TSP and (b)PM10 obtained trought SEM 